\title{
La importancia de las estrategias de afrontamiento en el bienestar psicológico en una muestra escolarizada de adolescentes
}

\section{The importance of coping strategies on psychological well- being in a school-based sample of adolescent population}

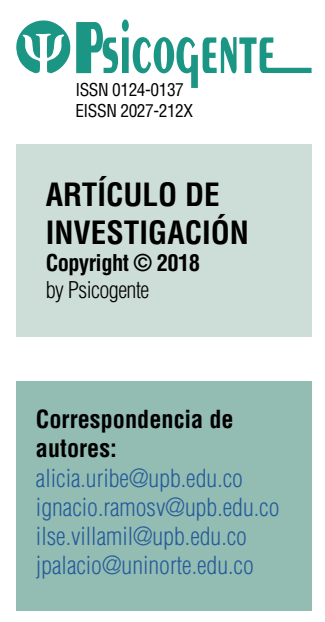

Recibido: 22-08-17 Aceptado: $11-01-18$ Publicado: 01-07-18

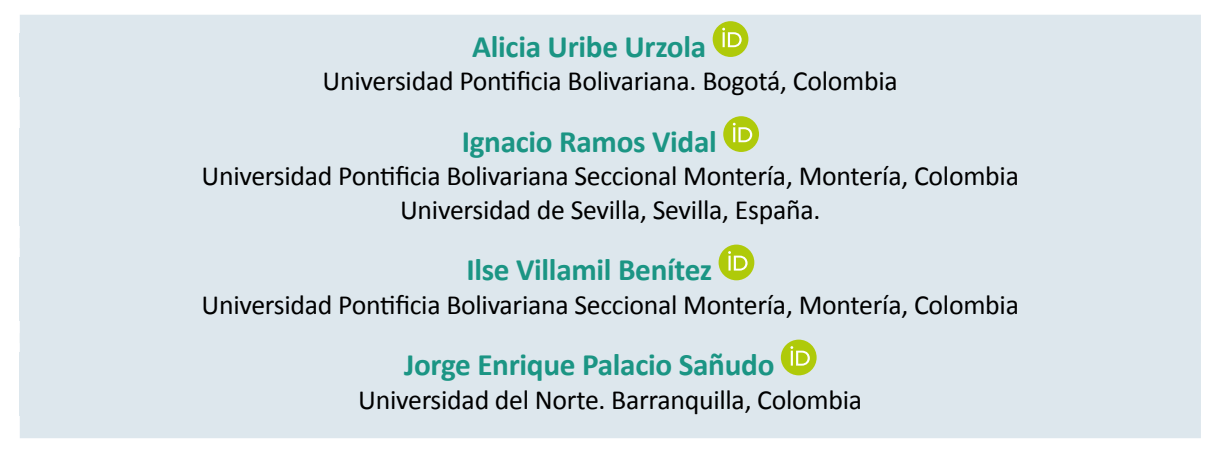

Resumen

Objetivo: Esta investigación tuvo como propósito identificar la relación entre las estrategias de afrontamiento y el bienestar psicológico.

Método: Se empleó un diseño transversal correlacional en una muestra de 104 adolescentes que estudian en colegios públicos de la ciudad de Montería (Córdoba, Colombia). Se aplicó la Escala de Afrontamiento para Adolescentes y el cuestionario de Bienestar Psicológico.

Resultados: Se identificó una asociación significativa entre las variables de afrontamiento y bienestar psicológico.

Conclusión: Los adolescentes que utilizan de forma recurrente estrategias de afrontamiento dirigidas a la resolución de problemas y a la búsqueda de apoyo social presentan mayores niveles de bienestar psicológico.

Palabras clave: adolescencia, afrontamiento, apoyo social, bienestar psicológico, colombia.

Abstract

Objective: This study aimed to identify the relationship between coping strategies and psychological well-being.

Method: A cross-correlation design was used in a sample of 104 adolescents studying in public schools in the city of Montería (Córdoba, Colombia). The Teen Coping Scale and the Psychological Wellbeing Questionnaire were applied.

Results: A significant association between the variables of coping and psychological well-being was identified.

Conclusion: Adolescents who use recurrent coping strategies aimed at solving problems and seeking social support present higher levels of psychological well-being.

Keywords: adolescence, coping, social support, psychological well-being, colombia.

Cómo citar este artículo (APA):

Uribe Urzola, A., Ramos Vidal, I., Villamil Benítez, I. \& Palacio Sañudo, J. E. (2018). La importancia de las estrategias de afrontamiento en el bienestar

psicológico en una muestra escolarizada de adolescentes. Psicogente 21(40), 440-457. https://doi.org/10.17081/psico.21.40.3082 


\section{INTRODUCCIÓN}

La adolescencia constituye una etapa crítica para el desarrollo madurativo y es considerada como una transición ecológica de vital importancia para comprender la adaptación del individuo al medio social (Bronfenbrenner, 2009). La adolescencia es un período clave para comprender el desarrollo en etapas posteriores del ciclo vital. En la investigación con población adolescente predominan los estudios centrados en (a) problemas comportamentales (b) la adopción de comportamientos prosociales y disruptivos; (c) en las relaciones dentro del grupo de iguales y (d) en los efectos de los vínculos paterno-filiales en la salud y en la adaptación psicológica (Steinberg, \& Morris, 2001).

La adolescencia es considerada una etapa en la que se pueden producir altos niveles de estrés debido al desempeño de nuevos roles psicosociales, a la toma de decisiones acerca del proyecto de vida y al enfrentamiento de los acontecimientos vitales tales como los cambios físicos, interpersonales, afectivos y cognitivos que vive el adolescente (Jessor, 1991; Wray-Lake, Syvertsen \& Flanagan, 2016). Al mismo tiempo durante la adolescencia el grupo de iguales tiende a suplantar de forma progresiva al círculo familiar como contexto primario de socialización, situación que en ocasiones puede llegar a producir un alto nivel de estrés en los adolescentes (Ramos-Vidal, 2016). En este sentido la evidencia empírica pone de relieve que el empleo de estrategias de afrontamiento pertinentes contribuye a mitigar los efectos negativos asociados al estrés que producen las nuevas demandas que deben enfrentar los jóvenes en esta etapa (Olsson, McGee, Nada-Raja, \& Williams, 2013; Cheng, Lau \& Chan, 2014). Estas demandas requieren que los adolescentes pongan en práctica estrategias de afrontamiento, es decir, que desplieguen comportamientos orientados a dar respuesta a las nuevas demandas que provienen tanto del propio proceso evolutivo, como del contexto social inmediato en el que el adolescente se desarrolla.

Al hablar de situaciones estresantes en la adolescencia Salotti (2006) dice que se deben tener en cuenta dos factores fundamentales: (i) Los acontecimientos vitales (cambios físicos, interpersonales, afectivos y cognitivos) que se pueden identificar como estresores; y (ii) Las características psicológicas (cognitivas, afectivas y comportamentales) que definen la adolescencia como tal, y que constituyen las variables individuales que determinan la valoración que el sujeto hace de los acontecimientos. 
Las situaciones que viven, sean producto de la transición hacia una nueva etapa vital, o situaciones inesperadas producto de eventos psicosociales, exigen a los adolescentes desarrollar gran parte del repertorio y estrategias con las que enfrentarán las diversas experiencias de la vida adulta. En la investigación realizada por Palacio y Romero (2009), los investigadores retoman una definición de los estilos de afrontamiento propuesta inicialmente por Lázarus y Folkman (1986) definiéndolos como las acciones y pensamientos que realiza el individuo para responder las exigencias tanto internas como externas. Por su parte González, Montoya, Casullo y Verdú (2002), consideran que la implementación de estrategias de afrontamiento en los adolescentes los prepara para tolerar, evitar o disminuir las consecuencias producidas por un evento estresante. En sentido inverso, las investigaciones señalan que la incapacidad para enfrentar los problemas mencionados es probable que conduzca a los adolescentes a experimentar malestar a través de síntomas psicosomáticos que de acuerdo a la literatura estarían también asociados a una tendencia pesimista de la vida (Martínez, Reyes, García \& González, 2006).

Los adolescentes, a través de las experiencias aprenden o desarrollan ciertas estrategias que poco a poco se van constituyendo en un estilo particular de afrontamiento, el cual a su vez, determinará el tipo de estrategias que deben utilizar en una situación específica. Cassaretto, Chau, Oblitas y Valdez (2003) señalan que "el estilo de afrontamiento puede ser entendido entonces como el conjunto de esfuerzos cognitivos y conductuales orientados a manejar (reducir, minimizar, dominar o tolerar) las demandas internas y externas de una determinada situación estresante" (p.369).

Otros autores sostienen (Compas, Mallaren \& Fondacaro, 1988; Frydemberg \& Lewis, 1997), que las estrategias pueden ser agrupadas de manera tal que conforman tres estilos de afrontamiento que representan aspectos funcionales (es decir aquellos que permiten superar de modo satisfactorio una situación potencialmente estresante), y aspectos disfuncionales (es decir, aquellas estrategias que pueden generar efectos negativos en la persona que pone en práctica dicha estrategia). Estas estrategias pueden sintetizarse en tres estilos: (a) estilos dirigidos a solucionar el problema de forma individual; (b) estilos de afrontamiento basados en la búsqueda de apoyo para hacer frente a la demanda que requiere el afrontamiento y (c) estilos improductivos que no suelen producir efectos positivos en el bienestar psicosocial de los adolescentes. El primer estilo consiste en trabajar de forma autónoma para resolver la situación problemática, manteniéndose optimista y relajado 
durante el proceso. Abarca las siguientes estrategias: concentrarse en resolver el problema, esforzarse y tener éxito, fijarse en lo positivo, diversiones relajantes y distracción física. El segundo estilo consiste en acercarse a otros para obtener apoyo social y abarca las siguientes estrategias: apoyo social, amigos íntimos, buscar pertenencia, acción social, apoyo espiritual y buscar apoyo profesional. Finalmente el estilo improductivo: se trata de una combinación de estrategias improductivas de evitación que muestra una incapacidad empírica para afrontar los problemas. Abarca las siguientes estrategias: preocuparse, hacerse ilusiones, no afrontamiento, reducción de tensión, ignorar el problema, auto-inculparse y reservarlo para sí.

El éxito o el fracaso al utilizar las estrategias de afrontamiento no implican necesariamente que existan unas estrategias mejores ni peores en sí mismas. Su efectividad se debe evaluar atendiendo a las posibilidades del entorno, los fines que se persiguen con determinada conducta, el momento vital del joven, entre otros factores asociados (Compas, Connor-Smith, Saltzman, Thomsen \& Wadsworth, 2001). Investigaciones acerca de la influencia del afrontamiento sobre la adaptación, como la descrita por Salotti (2006) revelan que las estrategias activas y orientadas hacia el problema moderan la influencia adversa de los sucesos de vida negativos sobre el funcionamiento psicológico. En una línea similar, Frydemberg y Lewis (1997) confirman a través de sus investigaciones, que las estrategias de afrontamiento pueden repercutir directamente sobre el estado de bienestar psicológico del sujeto. En un estudio reciente centrado en población adolescente de origen italiano, Cicognani (2011) muestra que el binomio conformado por (a) la disposición de fuentes de apoyo social y (b) el manejo efectivo de técnicas de afrontamiento pertinentes, determinan niveles óptimos de bienestar psicosocial en la población estudiada.

El bienestar psicológico es especialmente importante en la adolescencia, por tratarse de un período en el que se producen sustanciales transformaciones en la personalidad, al mismo tiempo que se define la identidad personal (y social) y se empieza a vislumbrar el proyecto de vida (Castro \& Sánchez, 2000; Casullo \& Fernández, 2001). Figueroa, Contini, Lacunza, Levín y Estévez (2005), en su investigación acerca de estrategias de afrontamiento y bienestar psicológico en población adolescente de bajo estrato socioeconómico y retomando la postura de Diener, Suh, \& Oishi (1997), aseveran que durante muchos años se relacionó el término bienestar con aspectos específicos como la apariencia física y la inteligencia. Así mismo, Lawton (1972) plantea que el bienestar es visto como una valoración cognitiva, como la 
evaluación de la congruencia entre las metas deseadas y las obtenidas en la vida.

Sin embargo, múltiples teóricos han afirmado que este término es mucho más complejo, ya que intervienen variables tanto a nivel individual como subjetivo. En este sentido, Ballesteros, Medina y Caicedo (2006, p.154), proponen una mirada distinta al bienestar psicológico, definiéndolo "como el esfuerzo por perfeccionarse y la realización del propio potencial". Así conceptualizado, el bienestar psicológico estaría estrechamente relacionado con tener un propósito en la vida, con un esfuerzo por afrontar las dificultades que se presenten y por conseguir las metas propuestas, mientras que el bienestar subjetivo está relacionado con sentimientos de relajación, ausencia de problemas y de presencia de sensaciones positivas (Villar, Triadó, Solé \& Osuna, 2003). Desde esta perspectiva, el bienestar psicológico centra su atención en el desarrollo de las capacidades y el crecimiento personal, concebidas ambas como los principales indicadores del funcionamiento positivo (Blanco \& Valera 2007; Abello-Llanos, Macías, Blanco-Abarca, MadariagaOrozco, Manrique-Palacio, Martínez-González \& Díaz-Méndez, 2009).

En otra propuesta, Ballesteros, Medina y Caicedo (2006), sugieren un modelo multidimensional según el cual, el bienestar psicológico se desarrolla durante toda la vida, y está dirigido a la perfección, que representa el desarrollo del verdadero potencial de cada individuo y se compone de seis dimensiones: auto-aceptación, relaciones positivas con otras personas, autonomía, dominio del entorno, propósito en la vida, y crecimiento personal. A continuación se describen brevemente cada una de estas dimensiones: (a) La auto-aceptación consiste en la capacidad que tiene una persona de sentirse satisfecho consigo misma. Esta dimensión suele ser el producto de una autoevaluación positiva de las propias características personales; (b) Relaciones inter-personales positivas: descrita como la facultad de mantener relaciones cordiales con otros, basadas en la confianza mutua y empatía; (c) La autonomía: esta dimensión hace referencia a la autodeterminación, independencia y regulación de la conducta. Las personas con autonomía son capaces de resistir en mayor medida la presión social y son capaces de adaptar su conducta a diferentes demandas del entorno; (d) Dominio del entorno: Las personas con un buen dominio del entorno poseen una mayor sensación de control sobre el mundo y se sienten capaces de influir sobre el contexto que les rodea (Díaz, Rodríguez, Moreno, Gallardo \& Dierendonk, 2005); (e) Propósito en la vida: este factor alude a las metas y proyectos que otorgan sentido a la vida de las personas. Finalmente, (f) el crecimiento personal se conceptualiza como 
el esfuerzo constante del ser humano por desarrollar todas sus destrezas o capacidades con el fin de sentirse autorrealizado.

En cuanto a la relación entre bienestar psicológico y afrontamiento, Figueroa, Cohen, Lacunza \& Gronda (2005) señalan que las estrategias de afrontamiento pueden incidir en el bienestar psicológico. Estudios llevados a cabo sobre estas variables muestran que el nivel de bienestar psicológico se relaciona con las estrategias de afrontamiento por lo que se deduce que los adolescentes utilizan distintos tipos de estrategias dependiendo de su nivel de bienestar.

Las emociones positivas y los recursos psicológicos de los individuos se constituyen en fortalezas que protegen a las personas de diversas enfermedades, de la misma manera que el adecuado manejo de un evento estresante podría disminuir las emociones negativas y mantener o incidir en mayor nivel de bienestar psicológico (Figueroa, et al., 2005; Mikkelsen, 2009). Por otro lado, las investigaciones señalan que los adolescentes con niveles moderados de bienestar psicológico emplean estrategias de índole pasiva que pueden llegar a considerarse como disfuncionales. Entre estas estrategias destacan el auto-inculparse, no compartir con los miembros de la red de apoyo la incertidumbre asociada al problema, ignorar el problema o emplear estrategias de evitación.

El empleo recurrente de estrategias de afrontamiento de carácter disfuncional suele repercutir negativamente en el bienestar psicosocial de la población que recurre a ellas (Contini, Coronel, Levin, \& Estevez, 2003). Sin embargo el uso adecuado de estrategias de afrontamiento positivas, es decir, aquellas que la literatura reconoce como funcionales, permiten hacer frente a la situación de manera satisfactoria y suelen producir correlatos positivos en el bienestar psicosocial de quienes las adoptan. A la luz de la revisión de la literatura efectuada, los objetivos de esta investigación se centran en conocer la asociación que existe entre los dos constructos evaluados, para a continuación poder determinar el efecto que los diferentes tipos de estrategias de afrontamiento producen en el bienestar psicosocial de la población adolescente evaluada.

\section{MÉTODO}

\subsection{Diseño}

La investigación es de tipo correlacional, evaluando la relación existente entre el bienestar psicológico y las estrategias de afrontamiento en adolescentes, 
de sexo femenino y masculino, entre 15 y 18 años, escolarizados en cuatro escuelas públicas de la ciudad de Montería.

\subsection{Participantes}

La muestra estuvo conformada por 104 adolescentes, 57 de sexo femenino y 47 de sexo masculino, que cursan estudios en instituciones educativas ubicadas en zonas de bajos recursos y socialmente vulnerables de la ciudad de Montería (Córdoba-Colombia). Se realizó un muestreo intencional por conveniencia para seleccionar a los participantes en el estudio.

\subsection{Instrumentos}

\subsubsection{Bienestar Psicológico}

Para evaluar el bienestar psicológico se utilizó la Escala de Bienestar Psicológico propuesta originalmente por Ryff (1989) -Scales of Psychological Well-Being, SPWB- al objeto de poder evaluar las dimensiones teóricas del bienestar psicológico. La escala consta de 29 ítems, que evalúan las seis dimensiones en las que se ha operacionalizado el constructo. Estas dimensiones son: (a) la Auto-aceptación (que es evaluada a través de los ítems 1, 7, 17, 13 y 24); (b) las Relaciones Positivas (evaluada mediante los ítems 2, 8, 12 y 25); (c) la Autonomía (medida con los ítems 3, 4, 9, 18, 19 y 23); (d) el Dominio del entorno (examinada a través de los reactivos 5, 10, 14, 20 y 29); (e) el Crecimiento personal (examinado en los ítems 22, 26, 27 y 28) y por último (f) el Propósito en la vida (medido con los reactivos 6, 11, 15, 16 y 21). Los ítems que están formulados en negativo han sido invertidos en la base de datos analizada.

\subsubsection{Afrontamiento}

En cuanto a las estrategias de afrontamiento, se evaluó con la Escala de Afrontamiento para Adolescentes desarrollada por Frydemberg y Lewis (1997). La versión final del ACS está compuesta por 80 elementos, 79 de tipo cerrado y uno final abierto, agrupados en 18 escalas diferentes, cada una de las cuales se compone de entre 3 y 5 reactivos, que reflejan 18 estrategias distintas de afrontamiento. Excepto el último elemento, que pide al sujeto que describa sus conductas de afrontamiento diferentes a las citadas, cada elemento describe una conducta de afrontamiento específica y el sujeto contesta el cuestionario indicando si realiza o le ocurre la conducta descrita "con mucha frecuencia", "a menudo", "algunas veces", "raras veces" o "no le ocurre". Las respuestas puntúan respectivamente 5, 4, 3, 2 o 1 puntos. 
El cuestionario consta de dos formas: una Forma Específica que permite evaluar las respuestas a un problema particular citado por el sujeto o propuesto por el examinador. Sin embargo, también se ha observado que las estrategias de afrontamiento que un sujeto elige suelen ser estables con independencia de la naturaleza del problema, por lo que se ha elaborado una Forma General del instrumento que se refiere a cómo el sujeto afronta sus problemas en general. La Forma General contiene los mismos elementos que la Forma Específica aunque las instrucciones de aplicación varían ligeramente. La escala y las diferentes sub-escalas gozan de unas aceptables propiedades psicométricas según los parámetros de fiabilidad establecidos por DeVellis (2016); de igual modo la estructura factorial se corresponde con la consignada en los modelos teóricos en los que se fundamentan ambos constructos.

\subsection{Procedimiento}

Primero se realizó una revisión exhaustiva del estado del arte sobre las variables del estudio, y se procedió a elaborar el marco teórico. Luego se estableció contacto con las directivas de las Instituciones educativas ubicadas en tres barrios de Montería, donde se encuentra una concentración significativa de la población objeto de estudio, para darles a conocer el proyecto y concertar las condiciones de la aplicación de los instrumentos. Concedida la autorización para realizar la aplicación de los cuestionarios a la población objetivo, se acordaron los horarios y fechas en que se realizaría la aplicación calculada entre 60 y 70 minutos aproximadamente. A los participantes se les explicó los objetivos del proyecto y se les pidió su consentimiento, el cual deberían firmar y ser autorizados por sus padres o acudientes, luego de explicarles en qué consistía su participación, se les motivó en el sentido de ser sinceros en sus respuestas, garantizándoles la confidencialidad en la información suministrada. Una vez aplicados los cuestionarios, se procedió a sistematizar y analizar la información para luego realizar el informe final del proyecto.

\subsection{Análisis de datos}

Los análisis se hicieron por sexo, 57 participantes de sexo femenino (54.8\%) y 47 de sexo masculino (45.2 \%). Las comparaciones por sexo se hicieron mediante la prueba T-Student, y para las comparaciones entre los dos test, independientemente del sexo se realizó la correlación de Spearman. Luego se corrió un análisis de regresión lineal para identificar las variables que se relacio- 
naban mejor entre sí. El nivel de significancia asumido en estas pruebas fue de 0.05 .

\section{RESULTADOS}

A continuación se presentan, primero los resultados estadísticos de la aplicación de la Escala de Afrontamiento para Adolescentes (ACS), y la Escala de Bienestar Psicológico de Ryff (Scales of Psychological Well-Being, SPWB).

\subsection{Estrategias de Afrontamiento}

En la Tabla 1 se muestran las correlaciones significativas entre las estrategias de afrontamiento de los adolescentes. Se evidencia que la mayor correlación entre las estrategias de afrontamiento de los jóvenes del estudio, se da entre las estrategias Búsqueda de Apoyo Social, Ayuda Espiritual y Resolución de Problemas. Se observan múltiples correlaciones entre las variables examinadas en esta investigación. En el siguiente apartado abordamos el estudio de las estrategias de afrontamiento en función del sexo de los participantes.

Tabla 1.

Correlación de Spearman entre las variables de estudio

\begin{tabular}{|c|c|c|c|c|c|c|c|c|c|c|c|c|c|c|c|c|c|}
\hline $\begin{array}{c}\text { VARIA- } \\
\text { BLES }\end{array}$ & RP & ES & PR & Al & PE & HI & NA & RT & so & IP & CU & RE & $\mathrm{AE}$ & PO & AP & DR & FI \\
\hline As & $0.561^{* *}$ & $0.320^{* *}$ & $0.311^{* *}$ & $0.521^{* *}$ & $0.443^{* *}$ & 0.151 & -0.183 & 0.070 & $0.389^{* *}$ & -0.008 & -0.086 & $-0.194^{*}$ & $0.602^{* *}$ & $0.473^{* *}$ & $0.703^{* *}$ & $0.368^{* *}$ & $0.322^{* *}$ \\
\hline $\mathrm{Rp}$ & & $0.398^{* *}$ & $0.507^{* *}$ & $0.414^{* *}$ & $0.336^{* *}$ & 0.188 & -0.103 & -0.003 & $0.220^{*}$ & -0.022 & 0.092 & .017 & $0.510^{* *}$ & $0.485^{* *}$ & $0.475^{* *}$ & $0.285^{* *}$ & $0.289^{* *}$ \\
\hline Es & & & $0.414^{* *}$ & 0.146 & $0.382^{* *}$ & $0.214^{*}$ & -0.085 & -0.103 & 0.113 & 0.107 & -0.029 & -.049 & $0.414^{* *}$ & $0.512^{* *}$ & $0.229^{*}$ & 0.192 & $0.216^{*}$ \\
\hline $\operatorname{Pr}$ & & & & 0.098 & $0.313^{* *}$ & $0.287^{* *}$ & -0.044 & -0.001 & 0.168 & -0.085 & -0.124 & -.092 & $0.421^{* *}$ & $0.530^{* *}$ & $0.317^{* *}$ & $0.252^{* *}$ & $0.229^{*}$ \\
\hline $\mathrm{Ai}$ & & & & & $0.370^{* *}$ & $0.284^{* *}$ & 0.179 & $0.364^{* *}$ & $0.438^{* *}$ & $0.238^{*}$ & $0.259^{* *}$ & 0.101 & $0.280^{* *}$ & $0.249^{*}$ & $0.510^{* *}$ & $0.409^{* *}$ & $0.449^{* *}$ \\
\hline $\mathrm{Pe}$ & & & & & & $0.280^{* *}$ & -0.039 & 0.044 & $0.400^{* *}$ & 0.084 & 0.074 & 0.048 & $0.444^{* *}$ & $0.318^{* *}$ & $0.324^{* *}$ & $0.334^{* *}$ & $0.229^{*}$ \\
\hline $\mathrm{Fi}$ & & & & & & & $0.233^{*}$ & 0.184 & 0.169 & 0.025 & $0.225^{*}$ & 0.097 & $0.340^{* *}$ & $0.238^{*}$ & $0.244^{*}$ & $0.215^{*}$ & $0.243^{*}$ \\
\hline $\mathrm{Na}$ & & & & & & & & $0.463^{* *}$ & 0.160 & $0.275^{* *}$ & $0.396^{* *}$ & $0.368^{* *}$ & -0.016 & -0.161 & -0.119 & -0.005 & -0.087 \\
\hline Rt & & & & & & & & & $0.390^{* *}$ & $0.240^{*}$ & $0.391^{* *}$ & $0.235^{*}$ & 0.030 & -0.158 & 0.073 & $0.211^{*}$ & 0.058 \\
\hline So & & & & & & & & & & 0.170 & 0.142 & -0.018 & $0.320^{* *}$ & $0.202^{*}$ & $0.528^{* *}$ & $0.344^{* *}$ & $0.246^{*}$ \\
\hline Ip & & & & & & & & & & & 0.135 & $0.210^{*}$ & 0.133 & 0.007 & 0.120 & $0.297^{* *}$ & $0.201^{*}$ \\
\hline $\mathrm{Cu}$ & & & & & & & & & & & & $0.419^{* *}$ & 0.000 & $-0.239^{*}$ & -0.013 & 0.000 & 0.059 \\
\hline Re & & & & & & & & & & & & & -0.015 & $-0.221^{*}$ & $-0.209^{*}$ & 0.006 & 0.043 \\
\hline $\mathrm{Ae}$ & & & & & & & & & & & & & & $0.461^{* *}$ & $0.525^{* *}$ & $0.263^{* *}$ & $0.247^{*}$ \\
\hline Po & & & & & & & & & & & & & & & $0.4111^{* *}$ & $0.325^{* *}$ & $0.430^{* *}$ \\
\hline Ap & & & & & & & & & & & & & & & & $0.401^{* *}$ & $0.412^{* *}$ \\
\hline Dr & & & & & & & & & & & & & & & & & $0.400^{* *}$ \\
\hline
\end{tabular}

Nota: $* p<0.001 * * p<0.001$

\subsection{Estrategias de Afrontamiento y Sexo}

Se encontró diferencia estadísticamente significativa en las estrategias de afrontamiento Invertir en amigos íntimos (Ai), Falta de afrontamiento (Na), 
Ignorar el problema (Ip), auto-inculparse (Cu), y distracción física (Fi), siendo el puntaje medio de estas estrategias mayor en los hombres tal como puede observarse en la Tabla 2. Los datos indican que para la población masculina aplican las diferentes estrategias de afrontamiento de manera más recurrente en comparación con la población femenina.

Tabla 2.

Diferencia de medias en función del sexo en las estrategias de afrontamiento y el bienestar psicosocial

\begin{tabular}{ccccccccc}
\hline ESTRATEGIAS & SEXO & $\mathbf{N}$ & MEDIA & DT & T & P & $\begin{array}{c}\text { IC 95 \% } \\
\text { (INFERIOR) }\end{array}$ & $\begin{array}{c}\text { IC 95 \% } \\
\text { (SUPERIOR) }\end{array}$ \\
\hline \multirow{2}{*}{$\mathrm{Ai}$} & Hombre & 47 & 64.17 & 15.558 & 2.572 & $0.012^{*}$ & 1.853 & 14.347 \\
& Mujer & 57 & 56.07 & 16.327 & & & & \\
$\mathrm{Na}$ & Hombre & 47 & 43.74 & 12.069 & 2.394 & $0.019^{*}$ & 0.954 & 10.184 \\
& Mujer & 57 & 38.18 & 11.590 & & & & \\
$\mathrm{Ip}$ & Hombre & 47 & 47.02 & 15.903 & 2.052 & $0.031^{*}$ & 0.626 & 12.539 \\
& Mujer & 57 & 40.44 & 14.678 & & & & \\
$\mathrm{Cu}$ & Hombre & 47 & 50.96 & 16.864 & 3.104 & $0.002^{*}$ & 3.418 & 15.514 \\
& Mujer & 57 & 41.49 & 14.235 & & & & \\
$\mathrm{Fi}$ & Hombre & 47 & 73.43 & 18.455 & 3.374 & $0.001^{*}$ & 5.966 & 22.991 \\
\hline
\end{tabular}

Nota: ${ }^{*} p<0.05 ;{ }^{*} p<0.001$

\subsection{Bienestar Psicológico}

Todas las correlaciones de las dimensiones del Bienestar Psicológico son significativas, excepto Autonomía con Dominio del Entorno, como se observa en la Tabla 3. En cuanto a los puntajes de bienestar psicológico en relación al género, se encontró diferencia estadística en el Dominio del Entorno, Crecimiento personal y Bienestar (Tabla 4), siendo mayores estos puntajes en las mujeres, es decir, las jóvenes presentan un mayor Dominio del Entorno, mayor Crecimiento Personal y tienen un mayor Bienestar en comparación a los adolescentes de sexo masculino de la muestra.

Tabla 3.

Correlación de Spearman de Bienestar Psicológico

\begin{tabular}{ccccccc}
\hline VARIABLES & $\begin{array}{c}\text { RELACIONES } \\
\text { POSITIVAS }\end{array}$ & AUTONOMÍA & $\begin{array}{c}\text { DOMINIO DEL } \\
\text { ENTORNO }\end{array}$ & $\begin{array}{c}\text { CRECIMIENTO } \\
\text { PERSONAL }\end{array}$ & $\begin{array}{c}\text { PROPÓSITO EN } \\
\text { LA VIDA }\end{array}$ & BIENESTAR \\
\hline Autoaceptación & $0.462^{* *}$ & $0.356^{* *}$ & $0.430^{* *}$ & $0.342^{* *}$ & $0.538^{* *}$ & $0.773^{* *}$ \\
Relaciones positivas & & $0.284^{* *}$ & $0.254^{* *}$ & $0.326^{* *}$ & $0.402^{* *}$ & $0.676^{* *}$ \\
Autonomía & & & 0.131 & $0.250^{*}$ & $0.375^{* *}$ & $0.621^{* *}$ \\
Dominio del entorno & & & & $0.435^{* *}$ & $0.259^{* *}$ & $0.565^{* *}$ \\
Crecimiento personal & & & & & $0.338^{* *}$ & $0.608^{* *}$ \\
Propósito en la vida & & & & & & $0.742^{* *}$ \\
\hline
\end{tabular}

Nota: ${ }^{*} p<0.05 ;{ }^{* *} p<0.001$ 
Tabla 4.

Bienestar Psicológico y Sexo en los Adolescentes

\begin{tabular}{ccccc}
\hline VARIABLES & SEXO & N & MEDIA & DT \\
\hline \multirow{2}{*}{ Dominio del entorno } & Hombre & 47 & 19.68 & 3.440 \\
& Mujer & 57 & 22.84 & 3.575 \\
Crecimiento personal & Hombre & 47 & 16.38 & 4.137 \\
& Mujer & 57 & 19.00 & 3.591 \\
Bienestar & Hombre & 47 & 119.66 & 18.190 \\
& Mujer & 57 & 128.79 & 17.740 \\
\hline
\end{tabular}

\subsection{Estrategias de Afrontamiento y Bienestar Psicológico}

Como puede observarse en la Tabla 5, la mayoría de las correlaciones entre las estrategias de afrontamiento y las dimensiones del bienestar psicológico son negativas en los jóvenes del estudio, lo cual implica que aquellos jóvenes que utilizan más la estrategia, menor tiende a ser su nivel de bienestar. Estos resultados indican la estrecha asociación que existe entre las estrategias de afrontamiento, y el bienestar psicosocial que muestra la población evaluada en esta investigación.

\section{Tabla 5.}

Correlación de Spearman entre las Estrategias de Afrontamiento y Bienestar Psicológico

\begin{tabular}{|c|c|c|c|c|c|c|c|}
\hline VARIABLES & $\begin{array}{c}\text { AUTO-ACEPTA- } \\
\text { CIÓN }\end{array}$ & $\begin{array}{l}\text { RELACIONES } \\
\text { POSITIVAS }\end{array}$ & AUTONOMÍA & $\begin{array}{l}\text { DOMINIO DEL } \\
\text { ENTORNO }\end{array}$ & $\begin{array}{l}\text { CRECIMIENTO } \\
\text { PERSONAL }\end{array}$ & $\begin{array}{l}\text { PROPÓSITO EN } \\
\text { LA VIDA }\end{array}$ & BIENESTAR \\
\hline As & 0.179 & 0.169 & 0.178 & 0.043 & 0.101 & 0.179 & $0.210^{*}$ \\
\hline Es & $0.266^{* *}$ & 0.089 & 0.099 & 0.139 & 0.087 & $0.338^{* *}$ & $0.253^{* *}$ \\
\hline $\mathrm{Na}$ & -0.169 & -0.180 & $-0.233^{*}$ & $-0.214^{*}$ & $-0.245^{*}$ & $-0.206^{*}$ & $-0.277^{* *}$ \\
\hline Rt & -0.142 & -0.137 & $-0.198^{*}$ & -0.169 & -0.147 & -0.144 & $-0.232^{*}$ \\
\hline Ip & -0.052 & -0.089 & -0.056 & -0.156 & $-0.259^{* *}$ & -0.105 & -0.152 \\
\hline $\mathrm{Cu}$ & $-0.235^{*}$ & -0.008 & $-0.261^{* *}$ & -0.187 & $-0.268^{* *}$ & -0.147 & $-0.262^{* *}$ \\
\hline $\operatorname{Re}$ & -0.070 & -0.009 & $-0.351^{* *}$ & 0.015 & -0.126 & -0.121 & -0.151 \\
\hline Po & $0.285^{* *}$ & 0.083 & 0.139 & 0.153 & 0.063 & $0.233^{*}$ & $0.258^{* *}$ \\
\hline
\end{tabular}

Nota: ${ }^{*} \mathrm{p}<0.05 ;{ }^{*} \mathrm{p}<0.001$

De forma complementaria desarrollamos tres modelos de regresión lineal para conocer el efecto que produce en el bienestar psicológico el uso de las estrategias de afrontamiento orientadas a: (a) la resolución de problemas (p.ej., Fijarse en lo positivo); (b) las relaciones interpersonales (p.ej., Búsqueda de apoyo social); y (c) las consideradas como improductivas (p.ej., Auto-inculparse). Para efectuar este análisis creamos cuatro nuevas 
variables: la primera agrupa las cinco estrategias de afrontamiento dirigidas a la resolución de problemas; la segunda variable creada ad-hoc es el resultado de la agregación de las seis estrategias de afrontamiento fundamentadas en las relaciones con los demás; la tercera variable es la resultante de agregar las siete estrategias consideradas como improductivas; mientras que la cuarta variable, que actúa como dependiente en los tres modelos de regresión, aglutina todas las dimensiones que dan forma al constructo bienestar psicológico. Los modelos de regresión ofrecen información relevante para comprender el impacto divergente que produce la utilización de estrategias de afrontamiento centradas en la resolución de problemas, en las relaciones inter-personales e improductivas en el bienestar psicológico de la población adolescente evaluada.

Los resultados de la Tabla 6 muestran que el empleo de estrategias de afrontamiento centradas en la resolución de problemas no producen efectos positivos en el bienestar psicológico de los participantes $\left[\Delta R^{2}=0.009 ; b=0.138\right.$; $p<0.163$; (IC: 95 \%: -0.017; 0.97)]. La misma tendencia es mostrada en la tabla 7, donde se aprecia la exigua proporción de varianza explicada por las estrategias de afrontamiento basadas en las relaciones sociales $\left[\Delta R^{2}=0.000\right.$; $B=0.036 ; p<0.332$; (IC: 95 \%: -0.024; 0.072)]. En sentido inverso, los resultados expuestos en la Tabla 8 reflejan que el empleo recurrente de estrategias de afrontamiento improductivas caracterizadas por la evitación o por la falta de ajuste, generan un impacto marcadamente negativo en el bienestar psicológico $\left[\Delta R^{2}=0.102 ; b=-0.333 ; p<0.001\right.$; (IC: $95 \%$ : -0.195; -0.056)]. En este segundo caso, el uso frecuente de estrategias de afrontamiento negativas explica el $10 \%$ de la varianza en el bienestar psicológico.

Tabla 6.

Modelo de regresión lineal entre el Bienestar Psicológico y las Estrategias de Afrontamiento

\begin{tabular}{ccccccccc}
\hline ESTRATEGIAS DE AFRON- & \multicolumn{8}{c}{ BIENESTAR PSICOLÓGICO - VD } \\
\cline { 2 - 8 } TAMIENTO - VI & $\mathbf{R}^{\mathbf{2}}$ & $\mathbf{\Delta \mathbf { R } ^ { \mathbf { 2 } }}$ & $\mathbf{B}$ & $\mathbf{P *}$ & $\mathbf{T}$ & $\mathbf{E E}$ & $\mathbf{F}$ & $\mathbf{D W}$ \\
\hline Resolución de problemas & 0.019 & 0.009 & 0.138 & 0.163 & 1.405 & 18.345 & 1.973 & 1.671 \\
Relaciones inter-personales & 0.009 & 0.000 & 0.096 & 0.332 & 0.975 & 18.435 & 0.950 & 1.677 \\
Improductivas & 0.111 & 0.102 & -0.333 & 0.001 & -3.566 & 17.464 & 12.719 & 1.831 \\
\hline
\end{tabular}

Nota: ${ }^{*} p<0.001 ; \mathrm{EE}=$ Error de estimación; DW= Estadístico de Durbin-Watson.

\section{DISCUSIÓN}

En esta investigación se ha analizado la conexión que existe entre los diferentes tipos de estrategias de afrontamiento que aparecen referenciadas en la literatura, y el nivel de bienestar psicológico reportado por la población 
examinada, estableciendo distinciones en función del sexo de los participantes. Se encontró que existe una relación significativa y positiva entre las estrategias de afrontamiento y el bienestar psicológico en los adolescentes de ambos sexos. Las estrategias dirigidas a la resolución del problema a través de las acciones realizadas por el individuo, o buscando el soporte de los demás, se relacionan con alto bienestar, mientras que las estrategias pertenecientes al estilo improductivo, se correlacionan con bajo bienestar.

Los jóvenes del estudio muestran acciones dirigidas especialmente hacia la búsqueda de apoyo como una fórmula efectiva de enfrentar las situaciones cotidianas. Disponer de personas de confianza con quienes compartir las emociones, problemas o dificultades, escuchar sus opiniones o simplemente saberse escuchado y aceptado como persona, ha demostrado tener un fuerte impacto tanto en la autoestima como en la capacidad de la persona para afrontar adecuadamente situaciones estresantes (Musitu \& Cava, 2003). El apoyo social proporciona a los individuos herramientas para expresar sus sentimientos, lograr la identificación con otras personas en crisis similares, lo cual puede revertirse en una mejor salud mental y afrontamiento más adecuado de los eventos estresantes (Lázarus \& Folkman, 1986).

Por otra parte, la ayuda espiritual es una estrategia que sirve como apoyo emocional para muchas personas, y genera notables efectos positivos para la salud mental, por ejemplo, (a) disminuyendo la tensión causada por los diferentes eventos estresores, (b) aumentando la autoestima, y (c) facilitando el ajuste a las demandas del contexto. En definitiva, esta estrategia puede erigirse en una fuente de esperanza y hace que el individuo se sienta protegido ante las amenazas provenientes del contexto social (Di-Colloredo, Aparicio \& Moreno, 2007).

Los adolescentes de sexo masculino utilizan más estrategias del estilo improductivo (falta de afrontamiento, ignorar el problema y autoinculparse), y una estrategia perteneciente al estilo en Relación con los Demás (Amigos íntimos); las mujeres por su parte, tienden a Preocuparse más, a Buscar Pertenencia y a Reservar para Sí sus dificultades; resultados equivalentes fueron obtenidos por otras investigaciones (Mikkelsen, 2009). El establecimiento de una relación íntima con otros significativos y el compromiso personal que ello implica, sirve de contención al joven para afrontar exitosamente las situaciones de la vida cotidiana. Al respecto, diversas teorías sobre la adolescencia coinciden en señalar que se trata de una etapa de definición de la identidad 
y, en ese proceso el logro de la autonomía es un objetivo muy importante (Papalia, Wendkos, \& Duskin, 2005).

El adolescente se propone adquirir independencia de las figuras parentales $y$, en esa búsqueda el grupo de pares constituye una red de apoyo social esencial. De allí que este vínculo sea, probablemente, el más importante. La puesta en juego de los afectos positivos y negativos, la rivalidad, la competencia, la solidaridad, va a exigir estrategias de afrontamiento que si no son exitosas lo conducirán a la angustia. El empleo de esta estrategia señala que el joven ha aprendido que es más factible solucionar sus problemas con la ayuda de alguien que si se encontrara solo. Por lo tanto, estas estrategias estarían protegiendo su salud y bienestar.

Las dimensiones Auto-aceptación y Propósito en la Vida son las que más aportan al Bienestar en los adolescentes del estudio, implicando que mientras mejor se sientan los sujetos consigo mismos, incluso siendo conscientes de sus limitaciones (Díaz, et al., 2005), y mientras tengan metas y proyectos que les permitan dotar de sentido su vida (Ryff, 1989), mayores serán sus niveles de bienestar psicológico. De acuerdo a Casullo \& Fernández (2000), los proyectos personales se refieren a los planes, las acciones que las personas desean realizar proporcionando coherencia y dirección a la vida.

La correlación obtenida fue negativa en las siguientes estrategias: No Afrontamiento ( $\mathrm{Na})$, Reducción de la Tensión (Rt) y Autoinculparse ( $\mathrm{Cu}$ ), todas ellas pertenecientes al grupo de estrategias improductivas, lo cual evidencia que cuando los adolescentes se autorresponsabilizan y se ven a sí mismos como culpables de sus dificultades, presentan una mirada pesimista de la situación actual, tienden a ver más el lado negativo de las cosas y se sienten en general poco afortunados (Frydemberg, 1997); de igual manera, si el adolescente tiende a aislarse y a impedir que otras personas conozcan su preocupación, se sienten desafortunados, pesimistas y no realizan actividades que les ayude a disminuir el estrés.

Para conocer el efecto de la utilización de las tres categorías en las que pueden clasificarse las estrategias de afrontamiento (i.e., resolución de problemas, relaciones inter-personales e improductivas), examinamos a través de tres modelos de regresión lineal el efecto que cada tipo de estrategia produce en el bienestar psicológico de los participantes. Los resultados evidencian que los estilos de afrontamiento que podemos denominar positivos, es decir, los tendentes a la resolución de problemas y los que se basan en la activación 
de vínculos inter-personales, no producen impactos estadísticamente significativos en los niveles de bienestar psicológico. Este resultado es contrario a lo que cabría esperar teniendo en cuenta la evidencia empírica revisada en esta investigación. Este resultado puede explicarse parcialmente en que el instrumento empleado mide la frecuencia en el empleo de las estrategias de afrontamiento, pero no evalúa la efectividad -real o percibida- de las estrategias de afrontamiento. De modo que es probable que sea necesario incorporar algún indicador de efectividad de las estrategias de afrontamiento empleadas para hacer frente a una demanda, para establecer el efecto tanto de la frecuencia de utilización, como del potencial real de cada estrategia de resolución (Livingston, Barber, Rapaport, Knapp, Griffin, King \& Sampson, 2013).

Sin embargo, los resultados del tercer modelo de regresión parecen constatar que el empleo recurrente de estrategias de afrontamiento improductivas produce un efecto negativo en el bienestar psicológico de la población examinada. Este resultado se sitúa en la línea de estudios anteriores que demuestran que usar este tipo de estrategias desemboca en consecuencias negativas para la salud mental en población adolescente (Guerra, Pereda, Guilera \& Abad, 2016). Una explicación a este hecho puede encontrarse en que el mero empleo de estrategias de afrontamiento improductivas, puede ser una consecuencia misma del estado anímico del sujeto, es decir, es plausible que exista una relación bidireccional entre bienestar psicológico y el empleo de este tipo de estrategias consideradas des-adaptativas. En cualquier caso, considerando que nuestra investigación es de carácter transversal y exploratorio, no podemos establecer con claridad relaciones de causalidad entre sendas variables, si bien es posible que exista un efecto feedback a través del cual las personas con bajos niveles de bienestar psicológico tiendan a utilizar con más frecuencia estrategias poco efectivas, cuyos resultados a su vez contribuyan a mantener bajos niveles de bienestar psicosocial.

Finalmente, si bien este estudio se encuentra en coherencia con resultados provenientes de otros contextos, abre nuevas posibilidades a la comprensión de las condiciones bajo las cuales los diversos factores psicosociales permean la vida de las personas en contextos determinados. Esta comprensión se constituye en un eje articulador de propuestas de atención en las cuales la preparación para asumir los diversos retos desde el desarrollo y potenciación de estrategias de afrontamiento, sea la alternativa. 
Nota de autores: Principales resultados de la investigación de la Maestría en Psicología de la Universidad del Norte titulada: Bienestar psicológico y estrategias de afrontamiento en adolescentes desplazados y no desplazados por la violencia sociopolítica en el sur de Montería.

\section{REFERENCIAS}

Abello-Llanos, R., Macías, M. A., Blanco-Abarca, A., Madariaga-Orozco, C., Manrique-Palacio, K., Martínez-González, M. \& Díaz-Méndez, D. (2009). Bienestar y trauma en personas adultas desplazadas por la violencia política. Universitas Psychologica, 8(2), 455-470. Available in: http://www.scielo.org.co/pdf/rups/ v8n2/v8n2a13.pdf

Ballesteros, B., Medina, A. \& Caicedo, C. (2006). El bienestar psicológico definido por asistentes a un servicio de consulta psicológica en Bogotá, Colombia. Universitas Psycologica, 5(2), 239-258. Available in: http://www.redalyc.org/ pdf/647/64750204.pdf

Blanco, A. \& Valera, S. (2007). Los fundamentos de la intervención psicosocial. En A. Blanco, y J. Rodríguez Marín (Coords.) Intervención Psicosocial (pp. 3-44). Madrid: McGraw-Hill.

Bronfenbrenner, U. (2009). The ecology of human development. Harvard University Press.

Cassaretto, M., Chau, C., Oblitas, H. \& Valdez, N. (2003). Estrés y afrontamiento en estudiantes de Psicología. Revista de Psicologia, 21(2), 364-392. Available in: https://dialnet.unirioja.es/descarga/articulo/994031.pdf

Castro, A. \& Sanchez, M. (2000). Objetivos de vida y satisfacción autopercibida. Psicothema, 12(1), 87-92. Available in: http://www.psicothema.com/psicothema. asp?id=257

Casullo, M., y Fernández, M. (2001). Estrategias de afrontamiento en estudiantes adolescentes. Revista del Instituto de Investigaciones, 6(1), 25-49. https://doi. org/10.1590/S1984-02922010000100003

Casullo, M. \& Fernández, M. (2000). Evaluación del bienestar psicológico en estudiantes adolescentes Argentinos. Revista de Psicología, 18 (1), 35-68. Available in: http:// revistas.pucp.edu.pe/index.php/psicologia/article/view/6840/6974

Cheng, C., Lau, H. P. B. \& Chan, M. P. S. (2014). Coping flexibility and psychological adjustment to stressful life changes: A meta-analytic review. Psychological Bulletin, 140(6), 1582-1607. http://dx.doi.org/10.1037/a0037913

Cicognani, E. (2011). Coping strategies with minor stressors in adolescence: Relationships with social support, self-efficacy, and psychological well-being. Journal of Applied Social Psychology, 41(3), 559-578. https://doi.org/10.1 111/j.1559-1816.2011.00726.

Compas, B. E., Connor-Smith, J. K., Saltzman, H., Thomsen, A. H. \& Wadsworth, M. E. (2001). Coping with stress during childhood and adolescence: problems, progress, and potential in theory and research. Psychological Bulletin, 127(1), 87. https://doi.org/10.1037//0033-2909.127.1.87

Compas, B., Mallaren, V. \& Fondacaro, K. (1988). Coping with stressful events in older childrens and young adolescents. Journal of Consulting and Clinical Psychology, 56(3), 405-411. http://dx.doi.org/10.1037/0022-006X.56.3.405

Contini, N., Coronel, P., Levin, M. \& Estevez, A. ( 2003). Estrategias de afrontamiento y bienestar psicológico en adolescentes escolarizados de Tucumán. Revista de Psicología , 21 (1), 41-69. Available in: http://revistas.pucp.edu.pe/index.php/ psicologia/article/view/3723

DeVellis, R. F. (2016). Scale development: Theory and applications, 26. United States of America: Sage publications. 
Díaz, D., Rodríguez, R., Moreno, B., Gallardo, I. \& Dierendonk, D. (2005). Adaptación española Escalas de bienestar psicológico. Psicothema, 18(3), 572-577. Available in: http://www.crecimientopositivo.es/Materiales/well-being-psycothema.pdf

Di-colloredo, C., Aparicio Cruz, D. P. \& Moreno, J. (2007). Descripción de los estilos de afrontamiento en hombres y mujeres ante la situación de desplazamiento. Psychologia. Avances de la disciplina, 1(2), 125-156. Available in: http://www. redalyc.org/articulo.oa?id=297224996002

Diener, E., Suh, E. \& Oishi, S. (1997). Recent findings on subjective well-being. Indian Journal of Clinical Psychology, 24, 25-41. https://doi.org/10.4236/sm.2013.31015

Figueroa, M., Cohen, S., Lacunza, A. \& Gronda, M. (2005). Análisis de situaciones problemáticas autopercibidas por adolescentes. Ridep, 19(1), 89-103. Available in: http://www.redalyc.org/pdf/4596/459645450006.pdf

Figueroa, M., Contini, N., Lacunza, A., Levín, M. \& Estévez, S. (2005). Las estrategias de afrontamiento y su relación con el nivel de bienestar psicológico. Un estudio con adolescenes de nivel socioeconómico bajo de Tucumán, Argentina. Anales de Psicología,21(1), 66-72. Available in: http://www.redalyc.org/articulo. oa? id=16721108

Frydemberg, E. (1997). Adolescent coping: theoretical and research perspectives. Londres: Routledge.

Frydemberg, E. \& Lewis, R. (1997). ACS: Escalas de afrontamiento para adolescentes (Manual de adaptación española). Madrid: TEA.

García, V. \& González B. (2000). El bienestar psicológico. Su relación con otras categorías sociales. Revista Cubana de Medicina General Integral, 16(6), 586-592. Available in: http://scielo.sld.cu/scielo.php?script=sci_arttext\&pi$\mathrm{d}=\mathrm{S} 086421252000000600010 \& / \mathrm{ng}=\mathrm{es} \& \mathrm{nrm}=\mathrm{iso}$

González, B., Montoya, C., Casullo, M. \& Verdú, B. (2002 ). Relación entre estilos y estrategias de afrontamiento y bienestar psicológico en adolescentes. Psicothema, 14(2), 363-368. Available in: https://es.scribd.com/document/353183380/ Relacion-entre-estilos-y-estrategias-de-afrontamiento-y-bienestar-psicologico-en-adolescentes

Guerra, C., Pereda, N., Guilera, G. \& Abad, J. (2016). Internalizing symptoms and polyvictimization in a clinical sample of adolescents: The roles of social support and non-productive coping strategies. Child Abuse \& Neglect, 54, 57-65. https:// doi.org/10.1016/j.chiabu.2016.03.004

Jessor, R. (1991). Risk behavior in adolescence: A psychosocial framework for understanding and action. Journal of Adolescent Health, 12(8), 597-605. https://doi. org/10.1016/1054-139X(91)90007-K

Lawton, M.P. (1972). The dimensions of morale. In D. Kent, R. Kastenbaum, and S. Sherwood (Eds.) Research, planning, and action for the elderly. New York: Behavioral Publications.

Lázarus, R. \& Folkman, S. (1986). Estrés y Procesos Cognitivos. Barcelona: Martínez Roca.

Livingston, G., Barber, J., Rapaport, P., Knapp, M., Griffin, M., King, D. \& Sampson, E. L. (2013). Clinical effectiveness of a manual based coping strategy programme (START, STrAtegies for RelaTives) in promoting the mental health of carers of family members with dementia: pragmatic randomised controlled trial. BMJ, 347, 62-76. https://doi.org/10.1136/bmj.f6276

Martínez, A., Reyes, G., García, A. \& González, J. (2006). Optimismo/pesimismo disposicional y estrategias de afrontamiento. Psicothema, 18(1), 66-72. Available in: http://www.psicothema.com/pdf/3177.pdf

Mikkelsen, F. (2009). Satisfacción con la vida y estrategias de afrontamiento en un grupo de adolescentes de Lima. (Tesis para licenciatura en Psicología). Pontificia Universidad Católica del Perú, Perú. 
Musitu, G. \& Cava, M. (2003). El rol del apoyo social en el ajuste de los adolescentes. Intervención Psicosocial, 12(2), 179-192. Available in: https://www.uv.es/lisis/ mjesus/8cava.pdf

Olsson, C. A., McGee, R., Nada-Raja, S. \& Williams, S. M. (2013). A 32-year longitudinal study of child and adolescent pathways to well-being in adulthood. Journal of Happiness Studies, 14(3), 1069-1083. https://doi.org/10.1007/s10902-0129369-8

Palacio, J. \& Romero, F. (2009). Características de los Estilos de Afrontamiento en Jóvenes Estudiantes de Secundaria. Informes Psicológicos, 11(13), 65-87. Available in: https://revistas.upb.edu.co/index.php/informespsicologicos/article/ view/1214/1255

Papalia, D., Wendkos, S. \& Duskin, R. (2005). Psicologia del desarrollo, la infancia y adolescencia. México: McGraw-Hill.

Park, N. (2004). The role of subjective well-being in positive youth development. The Annals of the American Academy of Political and Social Science, 591(1), 25-39. https://doi.org/10.1177/0002716203260078

Ramos-Vidal, I. (2016). Popularidad y relaciones entre iguales en el aula: un estudio prospectivo. Psicología Educativa, 22(2), 113-124. https://doi.org/10.1016/j. pse.2015.12.001

Ryff, C. (1989). Happiness is everything, or is it? Explorations on the meaning of psychological well-being. Journal of Personality and Social Psychology, 57(6), 1069-1081. https://doi.org/10.1037/0022-3514.57.6.1069

Salotti, P. (2006). Estudio sobre estrategias de afrontamiento y bienestar psicológico en una muestra de adolescentes de Buenos Aires. (Tesis para optar la licenciatura en Psicología). Universidad de Belgrano, Buenos Aires.

Steinberg, L. \& Morris, A. S. (2001). Adolescent development. Annual Review of Psychology, 52(1), 83-110. https://doi.org/10.1146/annurev.psych.52.1.83

Villar, F., Triadó, C., Solé, C. \& Osuna, M.J. (2003). Bienestar, adaptación y envejecimiento: cuando la estabilidad significa cambio. Revista Multidisciplinar de Gerontología, 13, 152-162. Available in: http://www.ub.edu/dppsed/fvillar/principal/ pdf/2003_revista_multidisciplinar_adaptacion.pdf

Wray-Lake, L., Syvertsen, A. K. \& Flanagan, C. A. (2016). Developmental change in social responsibility during adolescence: An ecological perspective. Developmental Psychology, 52(1), 130-142. https://doi.org/10.1037/dev0000067

Esta obra está bajo: Creative commons attribution 4.0 international license. El beneficiario de la licencia tiene el derecho de copiar distribuir, exhibir y representar la obra y hacer obras derivadas siempre y cuando reconozca y cite la obra de la forma especificada por el autor o el licenciante. 